Roy A. Mimna, 520 Meadowland Drive, Hubbard, Ohio 44425, USA. e-mail:

mimna@aol.com

\title{
ERRATA: TYPICAL CONTINUOUS FUNCTIONS ARE NOT CHAOTIC IN THE SENSE OF DEVANEY
}

\begin{abstract}
The author gives corrected statements of results in [M] and a corrected proof of Theorem 1 of $[\mathrm{M}]$.
\end{abstract}

In this note, necessary changes, clarifications and corrections are given to the results of $[\mathrm{M}]$. Here we prove the result in Theorem $1[\mathrm{M}]$, which is renamed below as Lemma 1 , for compact $n$-cubes in Euclidean n-space. In the end, we obtain a stronger result in that the set of functions in $C$ which are not topologically transitive, is shown to be a dense open subset of $C$. We also obtain a new result in Theorem 5 below. Hereafter, instead of $C$, we let $C(K, K)$ denote the set of all continuous functions of the form $f: K \rightarrow K$, where $K$ is a compact $n$-cube in $E^{n}$, where $E^{n}$ denotes Euclidean $n$-space with the usual metric. That is, $K$ is the Cartesian product of $n$ compact intervals in the real line. We put the uniform topology on the function space $C(K, K)$. Then since the range space $K$ is metrizable, we have the supremum metric; so that for elements $f$ and $h$ in $C(K, K), D(f, h)=\sup \{d((f(x), h(x)): x \in K\}$. For any set $E, \mathrm{Cl}(E)$ will denote the closure of $E$, and $\partial E$ will denote the boundary of $E$.

Lemma 1. Let $K$ be a compact $n$-cube in Euclidean $n$-space $E^{n}$, with the usual metric d. Let $C(K, K)$ denote the set of all continuous functions of the form $f: K \rightarrow K$ with the supremum metric. Then there is a dense open subset $W$ in $C(K, K)$ such that every function $f$ in $W$ has the property that for some nonempty open subset $U \subset K, f(\mathrm{Cl}(U)) \subset U$.

Key Words: Devaney's chaos, sensitive dependence on initial conditions, typical continuous functions.

Mathematical Reviews subject classification: 26A18, 54H20

Received by the editors June 19, 2001 
Proof. To show that $W$ is open in $C(K, K)$, let $h$ be any function in $W$. Then for some open set $U \subset K, h(\mathrm{Cl}(U)) \subset U$, where $U \neq K$ and $U$ is not dense in $K$. Since $h(\mathrm{Cl}(U))$ and $\partial U$ are both compact subsets of $K$, there is a positive distance $\mu>0$ between them. Let $N_{\mu / 2}(h)$ be an $\mu / 2-$ neighborhood of $h$ in $C(K, K)$. Then for any $f$ in $N_{\mu / 2}(h), f(\mathrm{Cl}(U)) \subset U$. Hence, $W$ is open in $C(K, K)$.

To show that $W$ is dense in $C(K, K)$, fix $\epsilon>0$, and let $h$ be any element in $C(K, K)$, where $h$ is not necessarily an element of $W$. Since $K$ has the fixed-point property, $h$ has a fixed point, say $x_{0}$, in $K$. Since $h$ is uniformly continuous on $K$, there exists $\delta^{*}>0$ such that for all $x_{1}, x_{2}$ in $K, d\left(x_{1}, x_{2}\right)<$ $\delta^{*}$ implies that $d\left(h\left(x_{1}\right), h\left(x_{2}\right)\right)<\epsilon$. Choose $\delta>0$ such that $\delta \leq \delta^{*}$ and $\delta<\operatorname{diam}(K)$. Let $B\left(x_{0}, \delta / 2\right)=B_{0}$ be the open ball of radius $\delta / 2$ about $x_{0}$. Let $B\left(x_{0}, \delta\right)=B_{1}$ be the open ball of radius $\delta$ about $x_{0}$. We now define a continuous function $f: K \rightarrow K$ by

$$
f(x)= \begin{cases}x_{0} & \text { if } x \in \mathrm{Cl}\left(B_{0}\right) \\ (2-2 t) x_{0}+(2 t-1) h(x) & \text { if } x \in \mathrm{Cl}\left(B_{1}\right) \backslash B_{0} \\ h(x) & \text { if } x \in K \backslash B_{1}\end{cases}
$$

where $t$ is defined so that for any $x \in \mathrm{Cl}\left(B_{1}\right) \backslash B_{0}, t=\frac{d\left(x, x_{0}\right)}{\delta}$. Then $f \in$ $C(K, K)$, and $D(h, f)<\epsilon$, and by $B_{0}=U$, we have $f(\mathrm{Cl}(U)) \subset U$. Hence, $W$ is a dense open subset of $C(K, K)$.

Theorem 2. There exists a dense open subset $W$ in $C(K, K)$ such that every function in $W$ is not topologically transitive, and hence not chaotic in the sense of Devaney.

We now make the obvious changes and corrections in the statement of other results in $[\mathrm{M}]$. Theorem 6 of $[\mathrm{M}]$ is restated as follows.

Corollary 3. There exists a dense open subset $W$ in $C(K, K)$ such that every function in $W$ has an asymptotically stable set.

By application of the above results and Lemma 1 of [BC], we can combine Corollary 7, Lemma 8, and Theorem 10 into the following.

Corollary 4. There exists an dense open subset $W$ in $C(K, K)$ such that every function $f$ in $W$ has the property that $C R(f) \neq K$, where $C R(f)$ denotes the chain recurrent set of $f$.

We now let $C(I, I)$ denote the set of all continuous functions of the form $f: I \rightarrow I$, where $I$ is a compact interval in the real line. As noted in $[\mathrm{M}]$, 
the set of all functions in $C(I, I)$ which are Block-Coppel chaotic, comprise an open subset of $C(I, I)$. In [K], although different terminology is used, Kloeden essentially shows that the set of functions of the form $f: I \rightarrow I$ which are Block-Coppel chaotic, make up a dense subset of $C(I, I)$. It follows that the set of Block-Coppel chaotic functions in $C(I, I)$ is dense and open. In the next result, we let $P(I, I)$ denote the set of all polynomials on a compact interval $I$ in the real line. We now show that there is a dense open subset of all polynomials in $P(I, I)$ which are Block-Coppel chaotic, but not chaotic in the sense of Devaney.

Theorem 5. Let $P(I, I)$ denote the set of all polynomials on the compact interval $I$. There exists a dense open subset $S \subset P(I, I)$ such that each polynomial in $S$ is Block-Coppel chaotic but not chaotic in the sense of Devaney.

Proof. $P(I, I)$ is dense in $C(I, I)$. Since there is a dense open subset $W \subset$ $C(I, I)$ such that every function in $W$ is not chaotic in the sense of Devaney, in the relative topology on the function space $P(I, I)$, the set of polynomials which are not chaotic in the sense of Devaney, is dense and open in $P(I, I)$. Similarly, there is a dense open subset of Block-Coppel-chaotic polynomials in $P(I, I)$. Since the intersection of a finite number of dense open sets is again dense and open, the theorem is proved.

\section{References}

[ABL] S. J. Agronsky, A. M. Bruckner, and M. Laczkovich, Dynamics of typical continuous functions, J. London Math. Soc. 40(2) (1988) 227243.

[BC] L. S. Block and E. M. Coven, Maps of the interval with every point chain recurrent, Proc. Amer. Math. Soc. 98(3) (1986) 513-515.

[K] P. E. Kloeden, Chaotic difference equations are dense, Bull. Austral. Math. Soc. 15 (1976) 371-379.

[M] R.A. Mimna, Typical continuous functions are not chaotic in the sense of Devaney, Real Analysis Exchange 25(2) (1999-2000) 947-953. 
Roy A. Mimna 